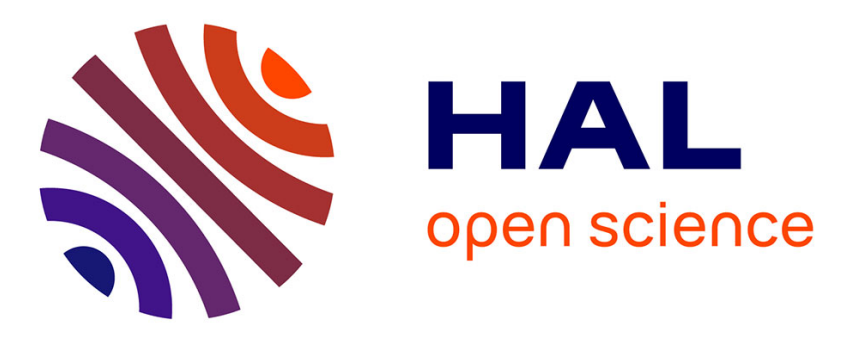

\title{
Evolution of the interaction of a new chemical entity, eflucimibe, with gamma-cyclodextrin during kneading process
}

A Gil, Alain Chamayou, E Leverd, J Bougaret, Michel Baron, G Couarraze

\section{To cite this version:}

A Gil, Alain Chamayou, E Leverd, J Bougaret, Michel Baron, et al.. Evolution of the interaction of a new chemical entity, eflucimibe, with gamma-cyclodextrin during kneading process. European Journal of Pharmaceutical Sciences, 2004, 23 (2), p.123-129. 10.1016/j.ejps.2004.06.002 . hal-01632796

\section{HAL Id: hal-01632796 \\ https://hal.science/hal-01632796}

Submitted on 8 Nov 2019

HAL is a multi-disciplinary open access archive for the deposit and dissemination of scientific research documents, whether they are published or not. The documents may come from teaching and research institutions in France or abroad, or from public or private research centers.
L'archive ouverte pluridisciplinaire HAL, est destinée au dépôt et à la diffusion de documents scientifiques de niveau recherche, publiés ou non, émanant des établissements d'enseignement et de recherche français ou étrangers, des laboratoires publics ou privés. 


\title{
Evolution of the interaction of a new chemical entity, eflucimibe, with $\gamma$-cyclodextrin during kneading process
}

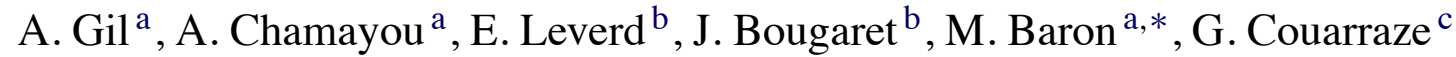 \\ a Department of Powders and Processes-UMR CNRS 2392, Ecole des Mines d'Albi, Campus Jarlard, 81013 Albi Cedex 09, France \\ ${ }^{\mathrm{b}}$ Department of Pharmaceutical Technology, Institut de Recherche Pierre Fabre, Rue Jean Rostand, 31319 Labège Innopole Cedex, France \\ ${ }^{c}$ Department of Pharmaceutical Physics-UMR CNRS 8612, Faculté de Pharmacie, Université Paris Sud, Rue Jean Baptiste Clément, \\ 92296 Chatenay Malabry Cedex, France
}

\begin{abstract}
The aim of this study was to enhance the low solubility of eflucimibe, a new chemical entity which can be used as medicine to treat dyslipidemiae, by complexation with $\gamma$-cyclodextrin. The complex was prepared using kneading method. The interaction evolution was studied during process by comparison of the semi-solid and physico-chemical states of the product. The evolution of the semi-solid state was followed by torque measurement when the evolution of physico-chemical state was studied by differential scanning calorimetry, infrared spectroscopy and by determination of the drug solubilisation profile. The interaction, which occurs during the process, is characterised by a modification of the product consistency and by a disappearance of the drug endothermic peaks, a disappearance of a drug spectral band and a widely improvement of the drug solubilisation profile. Indeed, after complete interaction, the drug quantity solubilised in specific conditions increased about 44-fold compared to those of untreated drug. Moreover, the comparison of the physico-chemical and semi-solid states during kneading process clearly shows that when the interaction takes place, a solidification of the paste occurs. The results of these works suggested that the formation of new solid phase allows an enhancement of the solubility of eflucimibe.
\end{abstract}

Keywords: Cyclodextrin; Complex; Solubility; Kneading; Semi-solid state

\section{Introduction}

New chemical entities are more and more poorly soluble in water (Lipinski, 2001, 2002). As a consequence, these compounds are not easy to develop as drug products using traditional formulation methods. Different formulation approaches (Panchagnula and Thomas, 2000) are generally used to overcome these difficulties: generation of salts (Berge et al., 1977); micronization (Boulay, 1985); use of complexing agent such as cyclodextrins (Duchêne et al., 1985); nanosizing (Merisko-Liversidge et al., 2003); melt extrusion (Breitenbach, 2002), etc. One of the difficulties is to generate a stable formulation and to use a process compatible with a future scaling-up.

This paper will focus on the preparation of a complex between a new chemical entity, eflucimibe (Patoiseau et al.,

\footnotetext{
* Corresponding author. Tel.: +33-5-63-49-30-63; fax: $+33-5-63-49-30-25$

E-mail address: michel.baron@enstimac.fr (M. Baron).
}

1997), in the presence of $\gamma$-cyclodextrin, using a kneading method.

Cyclodextrins are cyclic oligosaccharides able to host in their cavity, which is prevailingly hydrophobic, molecules of various kinds, thus affecting the physical and physico-chemical properties (e.g. solubility, stability, ...) of the guest, and ultimately, in the case of drugs, its bioavailability and therapeutic efficiency (Loftsson and Brewster, 1996).

Eflucimibe $\left[S-2^{\prime}, 3^{\prime}, 5^{\prime}\right.$-trimethyl-4'-hydroxy- $\alpha$-dodecylthio-phenylacetanilide] is a dodecylthio-phenylacetanilide derivative (molecular weight $=469.73 \mathrm{~g} \mathrm{~mol}^{-1}$ ) whose schematic structure is reported in Fig. 1. This drug, which can be used as medicine especially to treat dyslipidemiae, is characterized by its very poor solubility in water. This causes interindividual variations of its low bioavailability. The complexation with cyclodextrins may be expected to overcome these disadvantages.

The present paper is orientated to the investigation of the complexation feasibility between eflucimibe and 


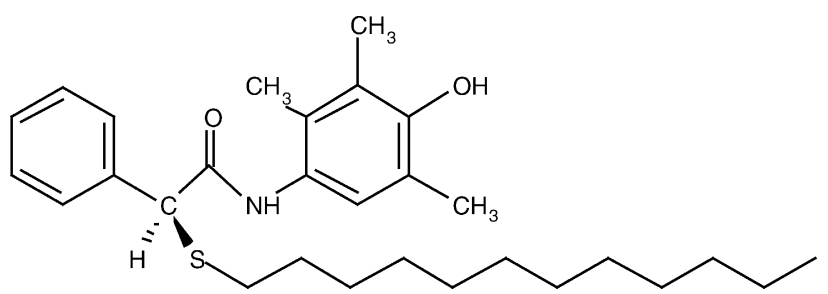

Fig. 1. Schematic structure of eflucimibe.

$\gamma$-cyclodextrin using the kneading method. The operating conditions implemented in the presented study have been optimised (Bougaret et al., 2002) and should be shortly published. In order to characterize interaction between active pharmaceutical ingredient and cyclodextrin, differential scanning calorimetry, infrared spectroscopy and determination of eflucimibe solubilisation kinetics were used.

\section{Materials and methods}

\subsection{Materials}

Eflucimibe was manufactured and supplied by Pierre Fabre Laboratories (Castres, France). $\gamma$-Cyclodextrin was purchased from Wacker-Chemie GmbH (München, Germany) and used as received, i.e. by taking into account water content.

\subsection{Preparation of binary systems}

Gamma-cyclodextrin was put in the Kneader bowl kept at $35^{\circ} \mathrm{C}$ and wet with purified water. The quantity of purified water added was calculated in order to obtain a mixture containing $25 \%$ of total water. After $5 \mathrm{~min}$, when the aqueous slurry of cyclodextrin was homogeneous, eflucimibe was added and the blend was kneaded thoroughly. The quantity of eflucimibe added was calculated in order to obtain an eflucimibe:cyclodextrin molar ratio of 1:2. For each sample taken, the paste obtained was dried at $40{ }^{\circ} \mathrm{C}$ for $12 \mathrm{~h}$ using a vacuum oven. The dried product was sieved below 300 mesh. The kneading device used was a Brabender rheometer with Banbury blades (Z-shaped). The rotation speed of the blades was fixed at $30 \mathrm{rpm}$. The viscosity of the system was followed during kneading step by the torque measurement following internal calibration.

\subsection{DSC and thermoanalytical procedure for the determination of the percentage of transformed eflucimibe $(Y)$}

Thermal analysis by DSC was carried out using a Perkin-Elmer DSC 7 apparatus. Samples of $3 \mathrm{mg}$ were put into aluminium pans. These pans were not sealed in order to permit the loss of gases evolved during the heating process. DSC scans were performed in triplicate under nitrogen, at a heating rate of $10^{\circ} \mathrm{C} \mathrm{min}^{-1}$ in the temperature range of $30-280^{\circ} \mathrm{C}$. Heats of fusion were automatically determined by the software following calibration with indium $\left(28.4 \mathrm{~J} \mathrm{~g}^{-1}\right)$, using integration of the areas under the DSC endothermic peaks of melting.

A thermoanalytical procedure can be applied to quantify the interaction yield (Giordano et al., 1992; Ginés et al., 1998). $F$ is the fraction by weight of eflucimibe in the starting mixture and $N$ is the fraction by weight of eflucimibe in the initial state after the kneading step. The percentage of transformed eflucimibe after interaction, $Y$, is calculated according to Eq. (1)

$Y=100-100\left(\frac{N}{F}\right)$

where $N$ is calculated according to DSC results and Eq. (2)

$N=\frac{\Delta H_{\text {eflucimibe melting after kneading step }}}{\Delta H_{\text {pure eflucimibe melting }}}$

\subsection{FTIR spectroscopy and spectroscopic procedure to follow eflucimibe interaction}

The infrared spectra were recorded on a Nicolet FTIR spectrometer. The analysed component was dispersed in $\mathrm{KBr}$ medium in solid state before acquisition.

For many drugs, the formation of a complex with cyclodextrin can lead to a modification of IR spectrum (Lin and Perng, 1992). As presented hereafter, eflucimibe interaction with cyclodextrin led to a decrease of eflucimibe band intensity. In order to quantify this modification, the Beer-Lambert law was applied by the determination of $\log$ $I_{0} / I$ with $I_{0}$ corresponding to the absorbance at $1572 \mathrm{~cm}^{-1}$ (spectral region where eflucimibe and cyclodextrin do not present spectral band) and $I$ corresponding to absorbance at $1537 \mathrm{~cm}^{-1}$ (spectral region where only eflucimibe presents spectral band).

\subsection{Determination of eflucimibe solubilisation kinetics}

The eflucimibe solubilisation kinetics were determined with samples corresponding to $50 \mathrm{mg}$ of eflucimibe. These samples were added to $100 \mathrm{ml}$ of the solubilisation medium corresponding to an aqueous solution containing $5 \%(\mathrm{w} / \mathrm{v})$ of sodium lauryl sulfate. The samples were continuously stirred while remaining in a water bath at $37^{\circ} \mathrm{C}$. At various time intervals, samples were withdrawn and filtered through $0.45 \mu \mathrm{m}$ membrane. The amount of eflucimibe dissolved was determined by HPLC using UV detection at $220 \mathrm{~nm}$. Acetonitrile and purified water at 82:18 (v/v) was run at $1 \mathrm{ml} \mathrm{min}^{-1}$ flow rate through a reverse-phase C8 column. 


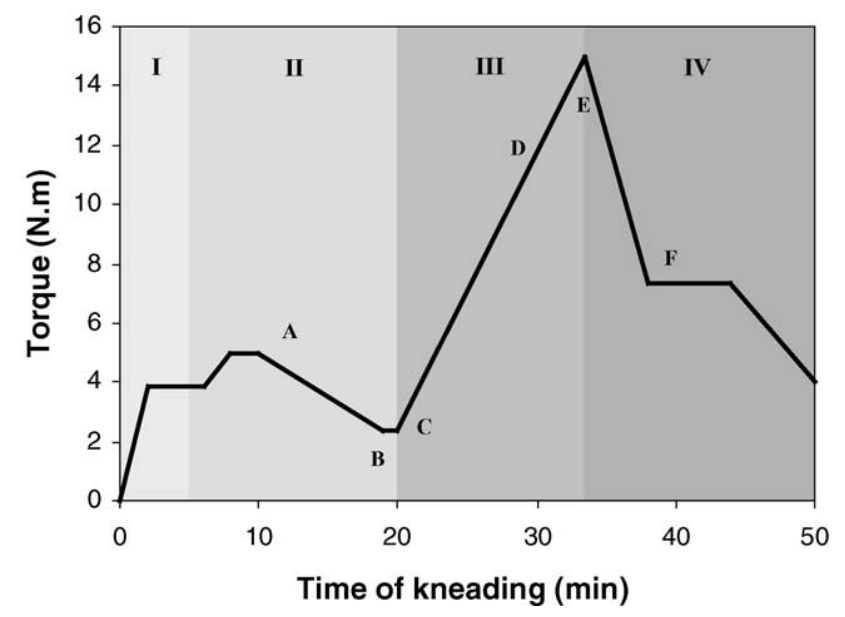

Fig. 2. Evolution of the torque applied during kneading of eflucimibe: $\gamma$-cyclodextrin aqueous mixture.

\section{Results and discussion}

\subsection{Evolution of product semi-solid state during kneading process}

The evolution of the torque applied in order to keep constant the rotation speed of the blades during the kneading process is presented in Fig. 2. As the torque is directly linked to the product viscosity, this evolution shows that the product semi-solid state changes during the process.

This evolution may be divided into four steps. The first one (I, 0-5 min) corresponds to the homogeneisation of aqueous $\gamma$-cyclodextrin slurry. The second begins at the time of eflucimibe addition. Eflucimibe was added to the mixture within approximately $1 \mathrm{~min}$. This second step (II, 5-20 min), for which the torque slightly decreases, corresponds to the preparation and the homogeneisation of the aqueous eflucimibe: $\gamma$-cyclodextrin mixture. Then, the third (III, 20-33 min) is characterised by a sharply increase of the product viscosity, corresponding to a solidification of the semi-solid form. The fourth (IV, 33-50 min) begins to the apex of the torque curve and is characterised by a large decrease of torque. It may be attributed to the breaking-up of the product after solidification.

During the kneading process, several samples (referenced A-F) have been taken (Fig. 2) and have been characterised by determination of water content, DSC, FTIR and by determination of eflucimibe solubilisation kinetics.

\subsection{Evolution of product physico-chemical state during kneading process}

In order to verify if the torque increase observed during kneading process was due to water evaporation or not, the determination of water content using Karl Fischer method was performed in samples taken before and after solidification (samples A and F, respectively). The results obtained,

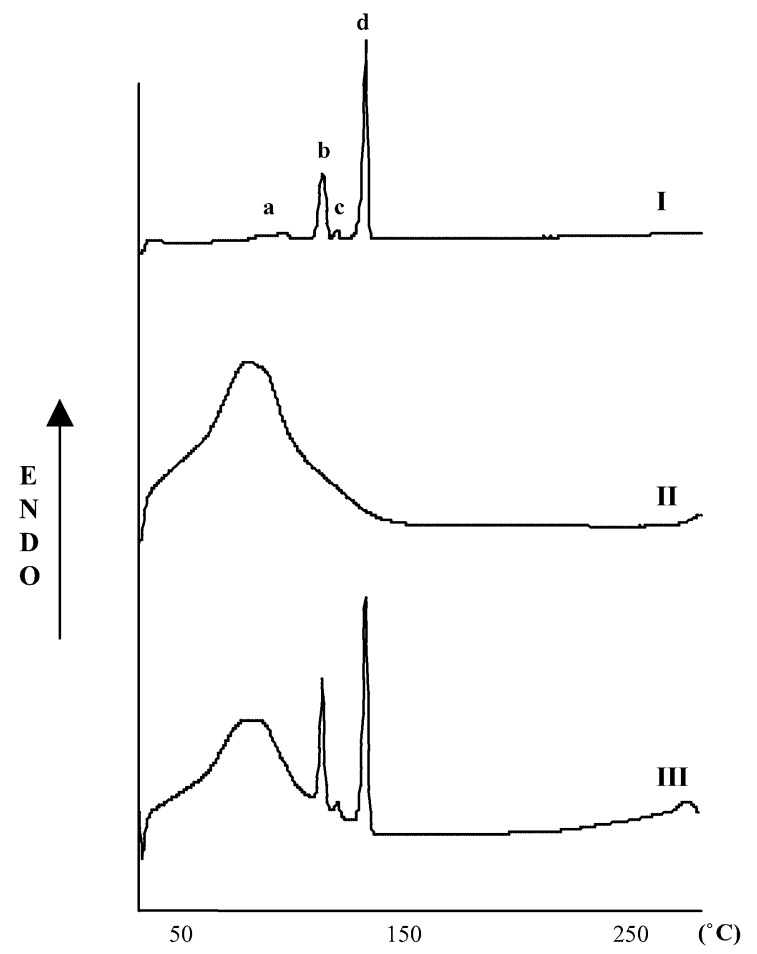

Fig. 3. Differential scanning calorimetry curves of: (I) pure eflucimibe; (II) $\boldsymbol{\gamma}$-cyclodextrin; (III) 1:2 eflucimibe: $\boldsymbol{\gamma}$-cyclodextrin physical mixture.

$23.7 \%$ for sample A and $22.7 \%$ for sample $\mathrm{F}$, showed no significant change of water content during kneading. This result demonstrated that the modification of semi-solid state during kneading was not due to a loss of water.

The thermal profiles of eflucimibe, $\gamma$-cyclodextrin and eflucimibe: $\gamma$-cyclodextrin physical mixture are reported in Fig. 3.

The eflucimibe DSC curve exhibits four endothermic peaks described in Table 1.

The thermal behavior of $\gamma$-cyclodextrin is characterised by a broad endotherm in the $50-130^{\circ} \mathrm{C}$ range, which can be attributed to water loss. The DSC profile of the 1:2 eflucimibe: $\gamma$-cyclodextrin physical mixture corresponds to a stacking of endotherms of each constituent. Fig. 4 presents the evolution of the kneaded product thermal behavior during kneading process.

Even if the interaction occurring during kneading process leads to a disappearance of all eflucimibe endotherms, the thermoanalytical procedure was performed considering only

Table 1

Characteristics of eflucimibe endothermic peaks

\begin{tabular}{llcc}
\hline Endotherm & Attribution & \multicolumn{2}{l}{ Peak characteristics } \\
\cline { 3 - 4 } & & $T_{\text {ONSET }}\left({ }^{\circ} \mathrm{C}\right)$ & $\Delta H\left(\mathrm{~J} \mathrm{~g}^{-1}\right)$ \\
\hline a & Solid-solid transition & 78.4 & -11.5 \\
b & Solid-solid transition & 111.4 & -35.9 \\
c & Solid-solid transition & 117.7 & -3.1 \\
d & Melting & 129.6 & -76.4 \\
\hline
\end{tabular}




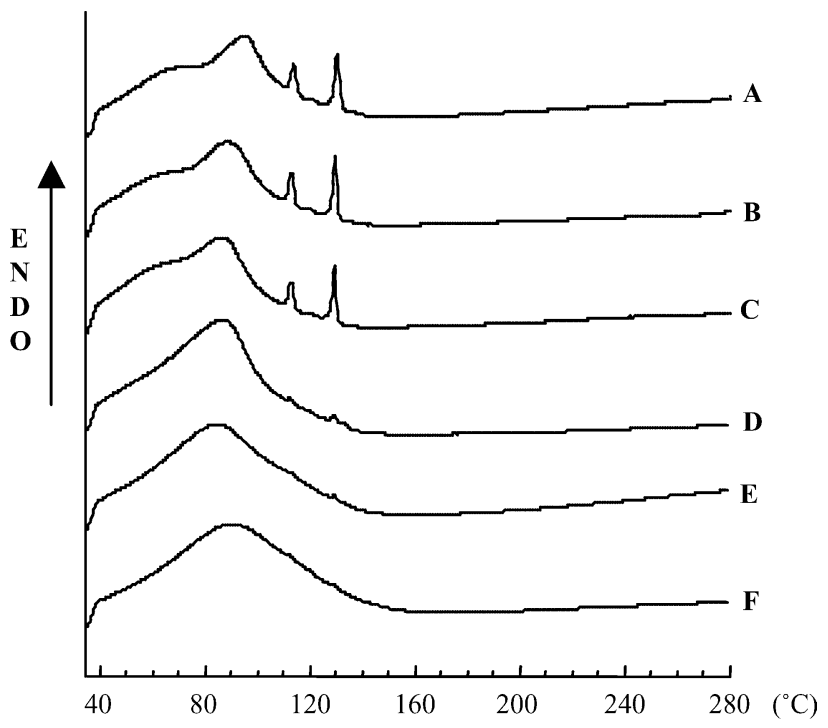

Fig. 4. Evolution of DSC curve during kneading process.

eflucimibe melting peak. The enthalpies relative to the melting event of eflucimibe were evaluated and the percentages of eflucimibe transformed during kneading were calculated and are presented in the Table 2. This characterisation shows that the interaction between eflucimibe and $\gamma$-cyclodextrin takes place during the solidification of the system and is optimal at the end of this one. Before the solidification, in the second step, the weak decrease in the enthalpy is probably due to the dispersion of eflucimibe within the slurry. After this one, in the fourth step, the transformation of eflucimibe by $\gamma$-cyclodextrin does not change; only the consistency of the mixture evolves.

Fig. 5 shows spectra of pure eflucimibe, $\gamma$-cyclodextrin and eflucimibe: $\gamma$-cyclodextrin physical mixture, which corresponds to superposition of IR absorption bands of each constituent. In the stretching region near $1540 \mathrm{~cm}^{-1}$, the $\gamma$-cyclodextrin poorly absorbs while eflucimibe exhibits an intense band located at $1537 \mathrm{~cm}^{-1}$ corresponding to $\mathrm{N}-\mathrm{H}$ bond. The spectroscopic procedure was performed according to the intensity of this band.

The evolution of the IR spectrum of the mixture during the kneading process is shown in Fig. 6.

For the samples taken during the second step, the IR spectrum of the mixture is similar to the corresponding physical

Table 2

Evolution of the enthalpy relative to the eflucimibe melting and of the percentage of eflucimibe transformed during kneading process

\begin{tabular}{lll}
\hline Sample & $\begin{array}{l}\Delta H_{\text {eflucimibe melting of kneaded product }} \\
\left(\mathrm{J} \mathrm{g}^{-1}\right)\end{array}$ & $\begin{array}{l}\text { Percentage of } \\
\text { transformed eflucimibe }\end{array}$ \\
\hline A & -8.4 & 20 \\
B & -8.1 & 25 \\
C & -6.6 & 39 \\
D & -1.7 & 85 \\
E & -0.5 & 96 \\
F & -0.2 & 99 \\
\hline
\end{tabular}

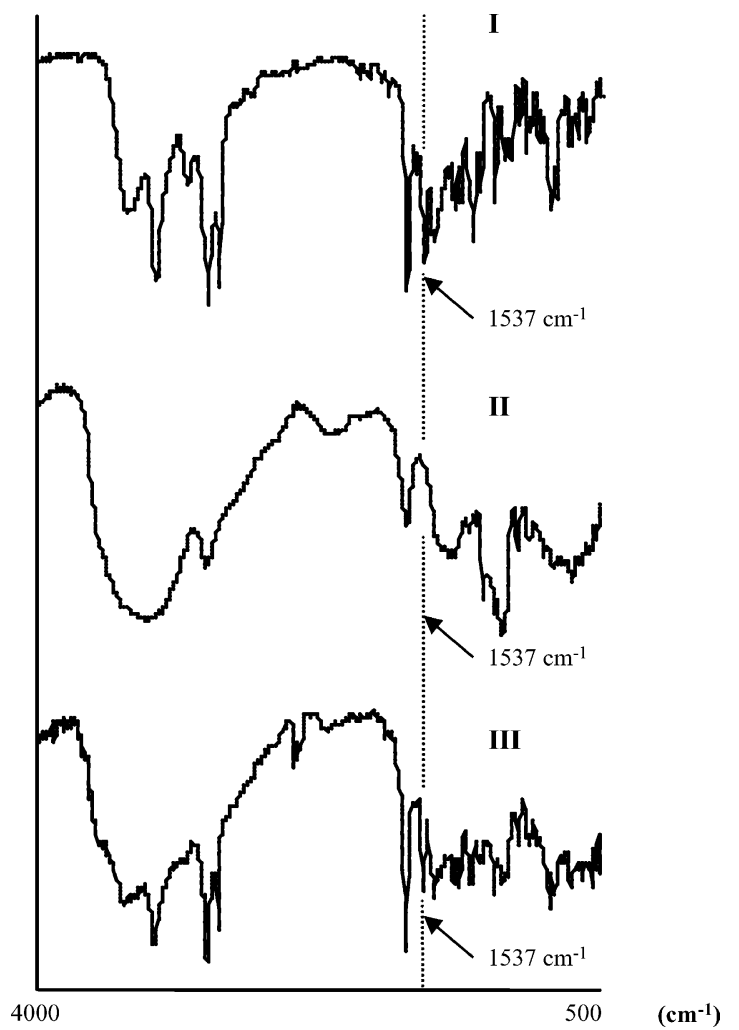

Fig. 5. FTIR spectra of: (I) pure eflucimibe; (II) $\gamma$-cyclodextrin; (III) 1:2 eflucimibe: $\gamma$-cyclodextrin physical mixture.

mixture (A and B). During the solidification, the IR spectra of the samples show a progressive decrease in the intensity of the eflucimibe band located at $1537 \mathrm{~cm}^{-1}$ (C and D) up to disappearance $(E)$. In the fourth step, the IR spectrum does

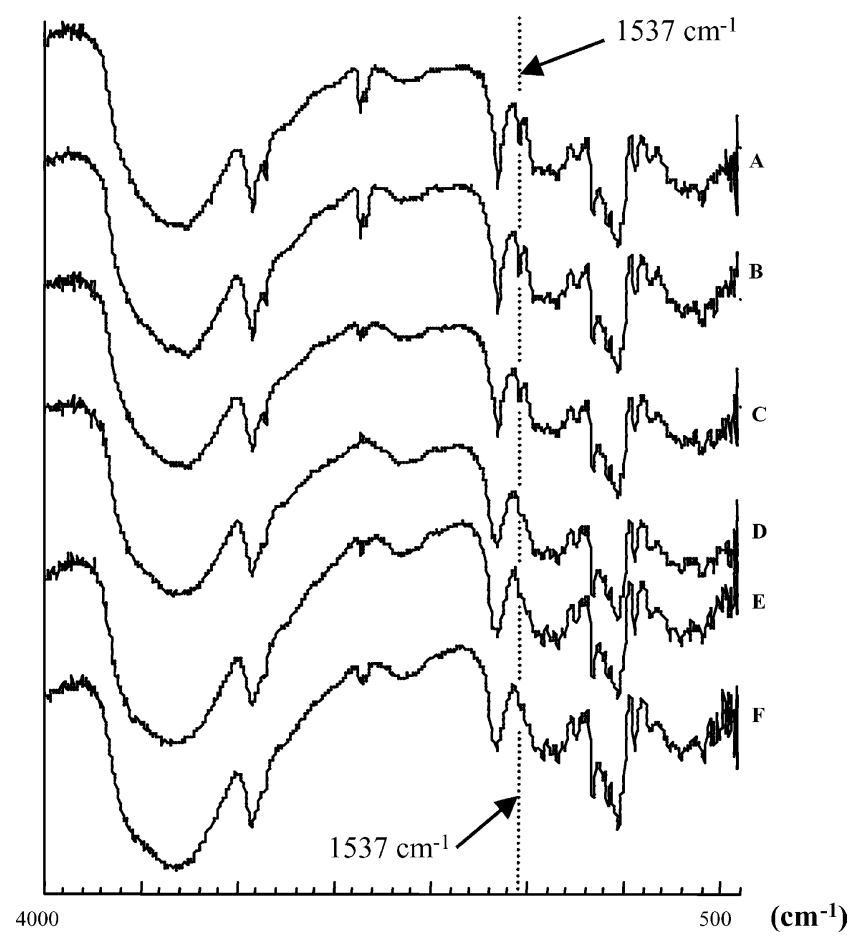

Fig. 6. Evolution of IR spectrum during kneading process. 
Table 3

Evolution of $\log I_{0} / I$ relative to eflucimibe band located at $1537 \mathrm{~cm}^{-1}$

\begin{tabular}{lllllll}
\hline Sample & A & B & C & D & E & F \\
\hline $\log I_{0} / I$ & 0.128 & 0.123 & 0.109 & 0.055 & 0.037 & 0.037 \\
\hline
\end{tabular}

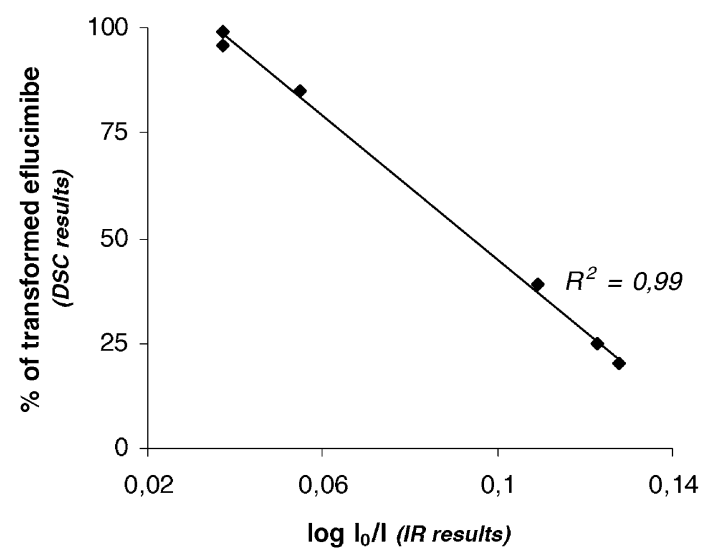

Fig. 7. Representation of DSC results in relation to IR results.

not change $(\mathrm{F})$. The Table 3 presents the evolution of $\log$ $I_{0} / I$, which is directly linked to the intensity of eflucimibe band located at $1537 \mathrm{~cm}^{-1}$.

These results confirm the previous ones obtained by DSC. Indeed, the quantitative evaluation of the interaction between eflucimibe and $\gamma$-cyclodextrin by IR shows that the interaction occurs during the solidification. Moreover, the representation presented in Fig. 7 of the percentage of transformed eflucimibe (DSC results) according to the $\log I_{0} / I$ (IR results) is a straight line that demonstrates the correlation between the characterisation methods implemented.

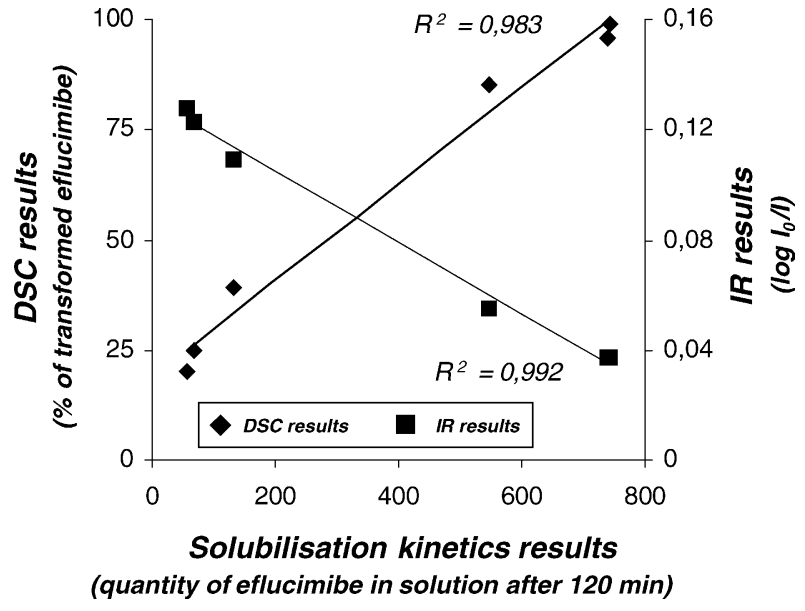

Fig. 9. Representation of DSC results and IR results in relation to solubilisation kinetics results.

The eflucimibe solubilisation kinetics were evaluated for all samples taken during the kneading process and are presented in Fig. 8.

The eflucimibe solubilisation kinetics are weakly improved compared to the corresponding physical mixture for the samples taken during the second step (A and B). For the samples taken during the solidification, in the third step, the eflucimibe solubilisation kinetics progressively improves. Indeed, the quantity of eflucimibe in solution after $120 \mathrm{~min}$ is increased by 8,32 and 43 times compared to the corresponding physical mixture for, respectively, the samples $\mathrm{C}$, $\mathrm{D}$ and E. For the sample taken after solidification $(\mathrm{F})$, the eflucimibe solubilisation kinetic is nearly the same as that of the sample taken to the apex of the viscosity curve (E). The quantity of eflucimibe in solution after $120 \mathrm{~min}$ showed a 44-fold increase compared to the physical mixture.

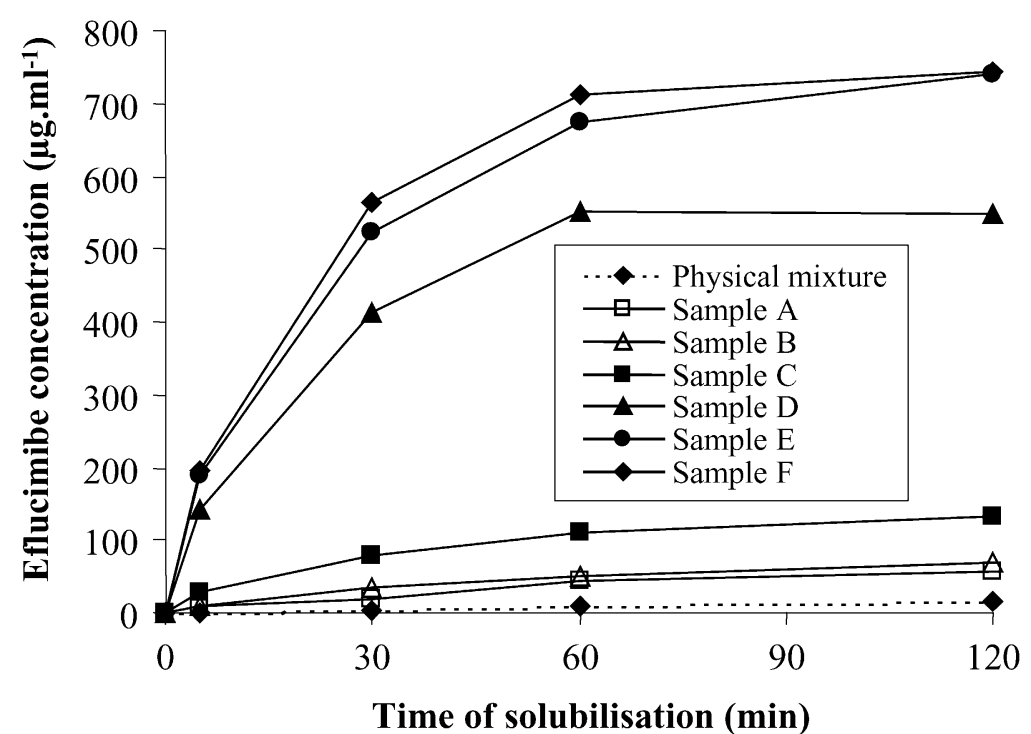

Fig. 8. Evolution of eflucimibe solubilisation kinetics during kneading process. 


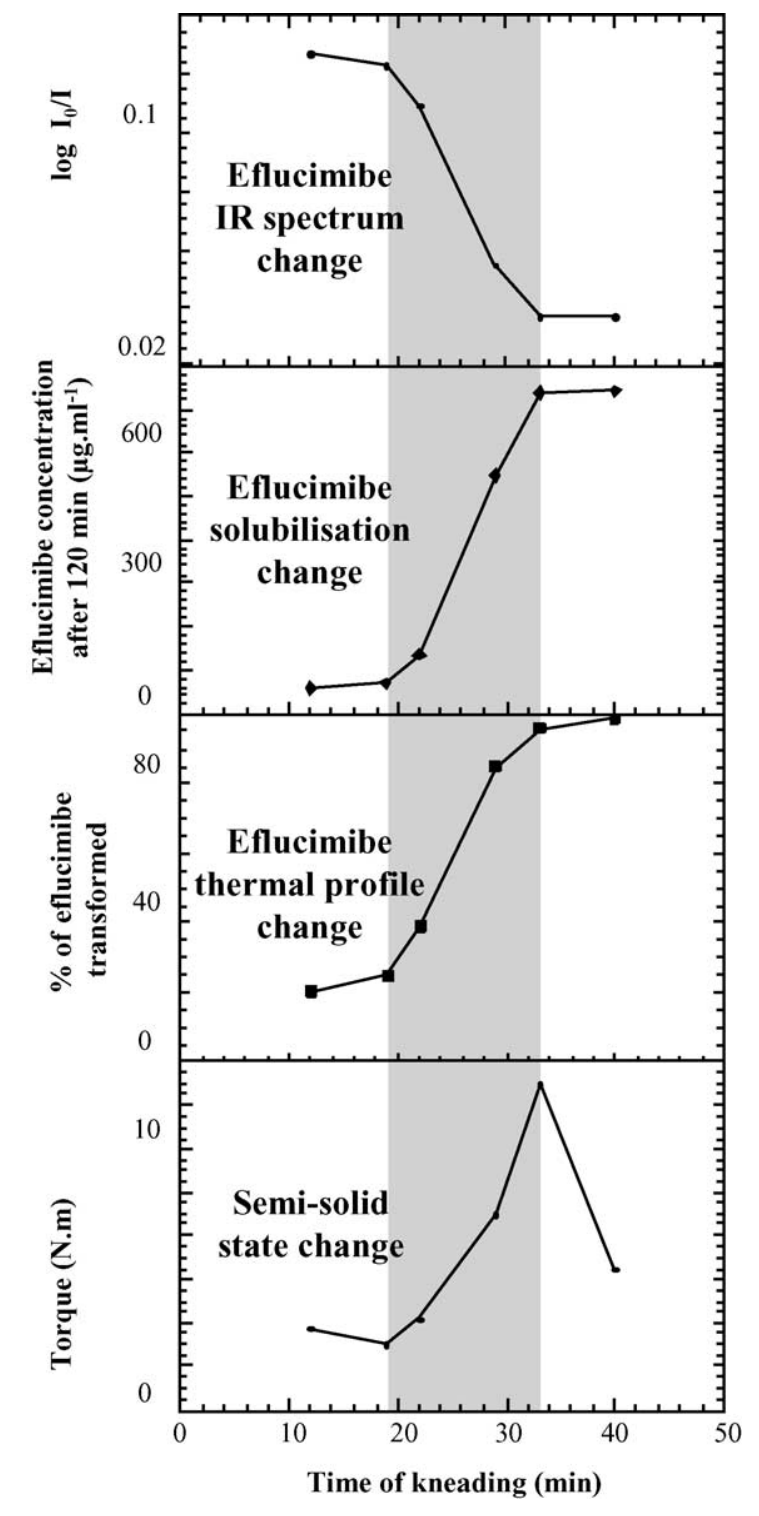

Fig. 10. Representation of the evolution of physico-chemical and semi-solid states of the eflucimibe: $\gamma$-cyclodextrin mixture during kneading process.

These confirm the results obtained by DSC and IR spectroscopy as shown in the Fig. 9.

\subsection{Comparison of physico-chemical and semi-solid states of the product during kneading process}

Fig. 10, which presents physico-chemical state in relation to semi-solid state of the product during kneading process, clearly shows that the molecular interaction occurring between eflucimibe and $\gamma$-cyclodextrin is directly linked to the solidification of the paste.

This study clearly shows that the complexation between eflucimibe and $\gamma$-cyclodextrin by kneading method corresponds to a reactive mixing, which may be divided into two successive main steps as schematized in Fig. 11. The first

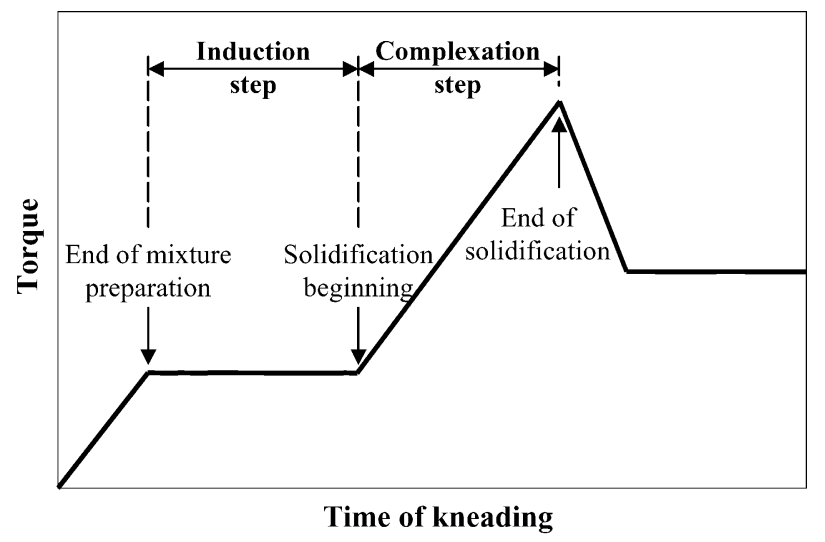

Fig. 11. Scheme of successive main steps occurring during kneading process.

one, which begins at the end of eflucimibe addition and finishes when solidification begins, corresponds to a step where the constituents remain in presence before expressing an interaction. This could be considered as an induction step. After that, from the start to the end of the solidification step, the formation of eflucimibe: $\gamma$-cyclodextrin complex occurs.

\section{Conclusion}

The formation of a complex between the hypolipidemic eflucimibe and $\gamma$-cyclodextrin has been studied by kneading method. In the solid state, using DSC, FTIR and the determination of eflucimibe solubilisation kinetics, the feasibility of a complex with $\gamma$-cyclodextrin has been shown. Moreover, the formation of eflucimibe: $\gamma$-cyclodextrin complex in a molar ratio 1:2 leads to a large improvement of eflucimibe solubilisation characteristics, which allowed the possibility that a formulation containing eflucimibe: $\gamma$-cyclodextrin complex obtained using kneading process should improve the eflucimibe bioavailability.

Finally, this study clearly shows the interest of using torque measurement to follow up the transformation of the product during the kneading process. Indeed, the solidification of the mixture can be used to monitor the interaction of eflucimibe with $\gamma$-cyclodextrin.

\section{References}

Berge, S.M., Bighley, L.D., Monkhouse, D.C., 1977. Pharmaceutical salts. J. Pharm. Sci. 66, 1-19.

Bougaret, J., Leverd, E., Ibarra, M-D., Gil, A., 2002. Anilide and cyclodextrin complexes, their preparation and their use as medicine in particular for treating dyslipidemiae. Patent WO 02/083632.

Boulay, G., 1985. Microbroyage et dissolution. S.T.P. Pharma. 1, 296-299.

Breitenbach, J., 2002. Melt extrusion: from process to drug delivery technology. Eur. J. Pharm. Biopharm. 54, 107-117.

Duchêne, D., Vaution, C., Glomot, F., 1985. La biodisponibilité des principes actifs par inclusion dans les cyclodextrines. S.T.P. Pharma. $1,323-332$. 
Ginés, J.M., Arias, M.J., Pérez-Martinez, J.I., Moyano, J.R., Morillo, E., Sanchez-Soto, P.J., 1998. Determination of the stoichiometry of 2,4 -dichlorophenoxyacetic acid- $\beta$-cyclodextrin complexes in solution and solid state. Thermochim. Acta 321, 53-58.

Giordano, F., Bruni, G., Bettinetti, G.P., 1992. Solid-state microcalorimetry on drug-cyclodextrin binary systems. J. Therm. Anal. 38, 26832691.

Lin, S-Y., Perng, R-I., 1992. Inclusion complex formation of acetominophen by heating and cogrinding with cyclodextrins. J. Incl. Phenom. Mol. Recognit. Chem. 14, 149-156.

Lipinski, C.A., 2001. Avoiding investment in doomed drugs, is poor solubility an industry wide problem? Curr. Drug Dis. 17-19.
Lipinski, C., 2002. Poor aqueous solubility-an industry wide problem in drug discovery. Am. Pharm. Rev. 5, 82-85.

Loftsson, T., Brewster, M.E., 1996. Pharmaceutical applications of cyclodextrins. J. Pharm. Sci. 85, 1017-1025.

Merisko-Liversidge, E., Liversidge, G.G., Cooper, E.R., 2003. Nanosizing: a formulation approach for poorly-water-soluble compounds. Eur. J. Pharm. Sci. 18, 113-120.

Panchagnula, R., Thomas, N.S., 2000. Biopharmaceutics and pharmacokinetics in drug research. Int. J. Pharm. 201, 131-150.

Patoiseau, J-F., Delhon, A., Junquero, D., Oms, P., Autin, J-M., 1997.2,3,5-trimethyl-4-hydroxyanilide derivatives, preparation thereof and therapeutical use thereof. Patent WO 97/19918. 